\title{
Implicaciones de los daños foliares sobre el balance final de carbono en diversos Quercus coexistiendo en un ambiente mediterráneo
}

\author{
García-Ciria, J., Jiménez, I., Gregorio, E., Rincón, P., González-Zurdo, P., Del Río, T., \\ Escudero, A., Mediavilla, S.*
}

Departamento de Ecología. Facultad de Biología. Universidad de Salamanca. 37071 Salamanca, España.

*Autor para correspondencia: ecomedv@usal.es

\section{Resumen}

Conocer el estado de nuestros bosques resulta indispensable para aplicar medidas preventivas y correctoras que puedan ser incorporadas a los planes de conservación. Pero también es crucial para la fiabilidad de los modelos destinados a estimar la capacidad real de secuestro de carbono de las masas forestales. La mayoría de dichos modelos asumen que la duración del aparato fotosintético coincide con la duración de las hojas individuales. Sin embargo, esto no es cierto cuando las hojas experimentan reducciones parciales de superficie por factores abióticos o por herbivoría. En este trabajo analizamos las pérdidas de superficie por ambos factores en hojas de cuatro especies de Quercus con distinta longevidad foliar, para tratar de estimar los costes en términos de pérdida de producción futura y sus implicaciones sobre la asimilación neta total de carbono de las hojas de las distintas especies de estudio. Nuestros resultados revelan que estos costes, aunque son siempre lo suficientemente importantes como para ser tenidos en cuenta, varían entre especies. Así, el descenso en la asimilación neta de carbono conseguida por las hojas de cada especie a lo largo de su vida debido a las pérdidas anticipadas de área por herbivoría resulta más bajo en las especies de mayor longevidad foliar. No obstante, mantener las hojas durante varios años conlleva un efecto desfavorable por lo que a los daños por factores climáticos se refiere, con pérdidas de asimilación neta de carbono por pérdidas de área que superan a las cifras obtenidas por herbivoría en las dos especies perennifolias para las que disponemos de datos de ambos tipos de daños. Nuestra conclusión, por tanto, es que las ventajas de una mayor longevidad foliar reduciendo el ataque de los herbívoros se ven contrarrestadas por el incremento en los daños que sufren las hojas a medida que se incrementa su duración y su exposición a los rigores climáticos.

Palabras clave: balance final de C, factores abióticos, herbivoría, longevidad foliar, Quercus. 


\section{Introducción}

La elaboración de modelos destinados a estimar el balance de carbono de las especies arbóreas constituye uno de los objetos prioritarios de estudio en ecología (Mediavilla and Escudero, 2003a; Kikuzawa and Lechowicz, 2006; Falster et al., 2011), porque resultan imprescindibles para comprender los patrones de distribución de las distintas especies y, fundamentalmente en los últimos años, por la fuerte influencia de la productividad vegetal sobre el control de la concentración atmosférica de $\mathrm{CO}_{2}$ (Dewar et al., 2009; Vaz et al., 2011). En este sentido, las distintas estrategias foliares desplegadas acaparan especial atención, porque las diferencias en las características de las hojas afectan fuertemente a los patrones de intercambio gaseoso (Aerts, 1995), de modo que los rasgos foliares específicos determinan el balance de carbono de los ecosistemas. Entre estos rasgos, la longevidad juega un papel primordial, porque representa el periodo de tiempo disponible para que la hoja ingrese a través de la fotosíntesis lo invertido en ella. Pero, además, puesto que una mayor duración impone a las hojas un refuerzo estructural orientado a garantizar su supervivencia, los cambios en longevidad se acompañan siempre de cambios en otra serie de características que determinan el rendimiento fotosintético (Warren and Adams, 2000; Takashima et al., 2004). Así, especies con menor longevidad foliar se acompañan de bajo peso por unidad de superficie (LMA), mayores concentraciones de nutrientes y menor concentración de fibras (Wright et al., 2004; Heberling and Fridley, 2012), lo que tiende a estar asociado con altas tasas de asimilación, si bien incrementa la vulnerabilidad frente a la herbivoría y a otros factores de estrés (Wright and Cannon, 2001; Shipley et al., 2006). Lógicamente, un mayor LMA conduce a las características opuestas, lo que se ve compensado por una mayor longevidad de los órganos vegetales (Warren and Adams, 2000; Takashima et al., 2004), por lo que un elevado LMA es un rasgo propio de las hojas perennes y de larga duración.

Hasta ahora se ha asumido que la menor asimilación instantánea de las hojas más longevas se compensa con el aumento de la duración de su vida productiva, de modo que, finalmente, especies con distinta longevidad foliar alcanzarían similar rendimiento, lo que permite su coexistencia en los mismos ambientes (Mediavilla and Escudero 2003a; Wright et al., 2004). Sin embargo, lo cierto es que en la mayoría de los casos en que se ha tratado esta cuestión se ha resuelto midiendo la fotosíntesis máxima obtenida por hojas jóvenes durante la época más favorable y multiplicándola directamente por la longevidad foliar (Reich et al., 1995; Mediavilla and Escudero 2003a; Kikuzawa and Lechowicz, 2006). Estos modelos asumen, por tanto, que la duración del aparato fotosintético coincide con la duración de las hojas individuales. Pero, evidentemente, esto puede no ser cierto cuando las hojas experimentan daños o reducciones parciales de superficie a lo largo de su vida a causa de estrés térmico u otros factores ambientales y particularmente a causa de la herbivoría. En este caso, la longevidad de las hojas individuales superaría a la de su masa, superficie o nutrientes, afectando estas pérdidas al balance final de carbono (Mediavilla and Escudero, 2003b). Especies con distinta longevidad foliar pueden variar en la intensidad de la herbivoría (Poorter et al., 2004; Matsuki and Koike, 2006), de 
modo que, si la herbivoría reduce el área foliar más en unas especies que en otras, esto puede repercutir de forma diferente en el rendimiento final a lo largo de la vida de sus hojas. Se hacen imprescindibles, entonces, estudios que aporten información al respecto que pueda ser incorporada a los modelos de balance de carbono para precisar las implicaciones de las diferencias en longevidad foliar.

Los ambientes de clima mediterráneo proporcionan un entorno idóneo para llevar a cabo este tipo de investigaciones porque cuentan con una gran diversidad de estrategias y hábitos foliares coexistiendo entre sí. En el presente trabajo nos proponemos analizar las pérdidas de superficie causadas por herbivoría y otros factores de éstres en hojas de cuatro especies arbóreas del género Quercus que, coexistiendo en un ambiente de clima mediterráneo, presentan hábitos foliares contrastados. El hecho de encontrarse en el mismo enclave nos permite descartar diferencias en los factores ambientales que pudieran condicionar diferencias en la intensidad del estrés al que se ven sometidas las hojas de las distintas especies o pudieran afectar a los ciclos de vida de los insectos que depredan las hojas y, por tanto, a la mayor o menor coincidencia de la aparición de herbívoros con la emergencia y nacimiento de las hojas, que es cuando se producen los ataques mayoritariamente. Incluimos, de este modo, únicamente las diferencias en las características de las hojas como factor condicionante de la intensidad de las pérdidas de área foliar. Nuestro objetivo es comprobar los efectos de la distinta longevidad foliar y los rasgos que confieren persistencia sobre la herbivoría y otros daños a las hojas, para, finalmente, tratar de analizar los costes en términos de pérdida de producción futura a causa de la pérdida prematura de área foliar y sus implicaciones sobre la asimilación neta total de carbono en las distintas especies.

\section{Material y métodos}

\subsection{Especies y zona de estudio}

El estudio se realizó en $Q$. faginea Lam. (quejigo), $Q$. pyrenaica Willd. (roble), Q. suber L. (alcornoque) y Q. ilex L. subsp ballota (Desf.) Samp. (encina). Las cuatro especies, ampliamente representadas en regiones de clima mediterráneo, se caracterizan por presentar hojas claramente diferenciadas en su duración media: desde en torno a 7 meses en el quejigo y roble, 14 meses en el alcornoque y algo superior a dos años en el caso de la encina. Representantes de todas ellas fueron seleccionados en la parcela de Valdelosa $\left(41^{\circ} 08^{\prime} 49.02^{\prime \prime} \mathrm{N}, 5^{\circ} 47^{\prime} 17.38^{\prime \prime} \mathrm{W}\right)$, ubicada en la zona norte de la provincia de Salamanca. El clima es mediterráneo frío, con una temperatura media anual de entre $11-12^{\circ} \mathrm{C}$ y una precipitación total anual que oscila entre los 350 y 500 $\mathrm{mm}$. El suelo es cambisol húmico, pasando en ciertas zonas a ser cambisol eútrico (Dorronsoro, 1992). La parcela es una típica zona de dehesa con ejemplares aislados (en torno a 50 especímenes ha $\mathrm{a}^{-1}$ y áreas de pasto entre ellos. Los árboles, individuos adultos en todos los casos, mostraban un diámetro del tronco (tomado a $1.3 \mathrm{~m}$ de altura) de entre 40 a $60 \mathrm{~cm}$ y una altura de entre 8-10 m. 


\subsection{Toma y procesamiento de las muestras}

Para cada especie seleccionamos al azar diez ejemplares, de cada uno de los cuales se extrajo una rama, a media altura de la copa. Todas las muestras se recolectaron a mediados de Octubre de 2013. Las ramas se transportaban al laboratorio, donde se separaban las distintas clases de edad presentes en las perennifolias. Todas las hojas se analizaban después visualmente para ser asignadas a distintas categorías según presentasen daños o no y el tipo de daño: hojas sanas, hojas atacadas por herbivoría, hojas con daños atribuibles a factores abióticos (estrés térmico, lumínico o hídrico) $\mathrm{y}$ hojas con ambos tipos de daños. Existen numerosos trabajos con abundante información gráfica, que permiten identificar los síntomas de estrés tanto bióticos como abióticos en las hojas (Vollenweider and Günthardt-Goerg, 2005; Günthardt-Goerg and Vollenweider, 2007). En las dos caducifolias, únicamente consideramos dos categorías (hojas sanas y atacadas por herbivoría), porque, en las fechas de recogida de las ramas, pudiera ser que algunas de las hojas mostrasen ya síntomas de senescencia, que erróneamente podríamos atribuir a daños por factores de estrés. Tras ser clasificadas, efectuamos el recuento de número de hojas en cada categoría, para cada uno de los 10 ejemplares seleccionados en cada especie. Finalmente un total de 15.825 hojas fueron inspeccionadas.

Cinco hojas de cada tipo en cada individuo se seleccionaban hasta un total de 50 por especie, clase de edad y categoría para analizar los siguientes rasgos. El espesor foliar se obtuvo con un calibre digital (Digimatic micrometer, Mitutoyo, Japan), como
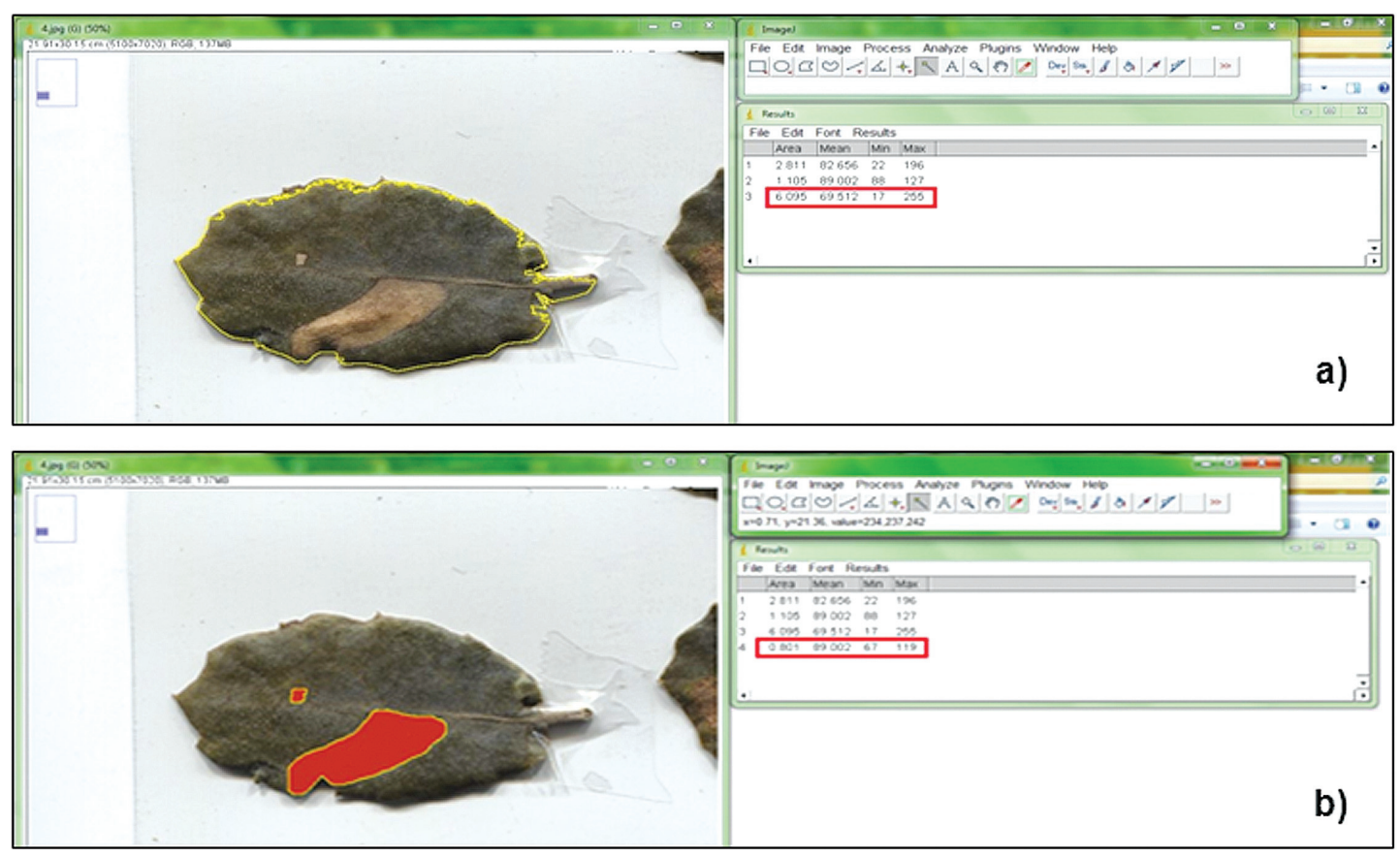

Figura 1. Captura de pantallas del programa ImageJ identificando el área foliar total (a) y el área dañada (b), en este caso por factores de estrés abiótico. 
media de tres medidas tomadas en posiciones al azar en cada hoja, evitando los nervios principales. El área total proyectada de cada hoja se determinó por medio de un analizador de imagen (Delta-T Devices LTD, Cambridge, UK). Las muestras eran posteriormente secadas a $70^{\circ} \mathrm{C}$ hasta peso constante y la masa por hoja determinada en una balanza analítica Sauter AR70. Con los datos así obtenidos, estimamos la masa por unidad de superficie (LMA) como cociente entre el peso y área proyecta de las hojas.

Las pérdidas de área foliar debidas a herbivoría o a daños por otros factores se analizaron también en 5 hojas por individuo hasta un total de 50 de cada especie y clase de edad, mediante el empleo del programa ImageJ (http://rsb.info.nih.gov/ij/; Abràmoff et al. 2004). Para ello, las hojas debidamente alineadas eran adheridas por el peciolo en transparencias y escaneadas a alta resolución. El programa ImageJ permite analizar estas imágenes, proporcionando tanto el área completa de cada hoja, como el área ausente debido a pérdidas por el ataque de herbívoros, y las áreas con una coloración distinta debida a daños por diversos factores de estrés (Fig. 1). La superficie perdida en los bordes de la hoja se estimó reconstruyendo el borde perdido y calculando la superficie ausente. De este modo, para cada una de las 50 hojas analizadas en cada caso pudo estimarse el porcentaje de superficie perdida bien por herbivoría o por otro tipo de daños.

\subsection{Análisis de los datos}

A partir del recuento de número de hojas, se estimó para cada especie y clase de edad, en cada árbol, el porcentaje de hojas sanas, así como el de hojas dañadas, tanto por herbivoría, como por otros factores. Un valor final para cada especie y clase de edad se obtuvo como promedio de los correspondientes a los 10 árboles seleccionados en cada caso. Por lo que se refiere a los distintos rasgos foliares analizados, obtenidos todos los datos, un valor medio para cada uno de ellos se estimó para las hojas de cada especie, clase de edad y categoría según el daño, como promedio de los valores correspondientes a las 50 hojas analizadas en cada caso. El análisis de la varianza de una vía (ANOVA) se empleó para examinar las posibles diferencias existentes entre nuestras especies en los distintos parámetros estimados. El análisis de regresión fue utilizado finalmente para comprobar las posibles relaciones entre los distintos parámetros de estudio. Todos los análisis estadísticos se llevaron a cabo empleando el programa SPSS (SPSS Inc., Chicago, IL, USA).

\section{Resultados}

\subsection{Niveles de herbivoría e intensidad de los daños por factores de estrés abiótico en las hojas de las distintas especies.}

Entre nuestras especies, se observan diferencias significativas en los niveles de daños causados por herbivoría que parecen estar relacionadas con las diferencias en 
longevidad foliar. Las caducifolias muestran tanto una proporción de hojas dañadas como de área media consumida por hoja significativamente superior a los de las dos perennifolias, entre las que los porcentajes más elevados le corresponden a las hojas de la especie con menor longevidad (Tab. 1). Ni en Q. ilex ni en Q. suber se aprecian, sin embargo, diferencias a nivel de las distintas clases de edad, mostrando todas ellas similares proporciones de hojas atacadas, así como de área consumida (Tab. 1). Contrariamente, en relación a los factores abióticos se observa un claro ascenso en proporción de hojas dañadas y fracción dañada por hoja entre las sucesivas cohortes (Tab. 1). Aun así, cuando comparamos las hojas de la misma cohorte de ambas especies, de nuevo es $Q$. suber la que muestra mayor intensidad de los daños, también por factores de estrés, que las de la misma cohorte de Q. ilex. Entre estas dos especies para las que disponemos de datos de los dos tipos de daños, se observa que la fracción de área perdida por hoja alcanza siempre valores muy superiores para los daños por factores abióticos en comparación al área perdida por herbivoría (Tab. 1).

Tabla 1. Porcentaje de hojas atacadas por herbivoría y de hojas dañadas por distintos factores abióticos (valor medio, $\mathrm{SE}$ entre paréntesis, $\mathrm{n}=10$ árboles por especie) y porcentaje de área media perdida por hoja en ambos casos en las distintas especies y clases de edad en las perennifolias. Letras distintas en la misma columna indican diferencias significativas entre tipos de hoja (especie y clase de edad) para $\mathrm{P}<0.05$.

\begin{tabular}{|c|c|c|c|c|c|c|c|}
\hline Especie & $\begin{array}{l}\text { Longevidad } \\
\text { foliar (días)* }\end{array}$ & $\begin{array}{l}\text { Edad } \\
\text { foliar }\end{array}$ & $\begin{array}{l}\mathrm{N}^{\circ} \text { hojas } \\
\text { contadas }\end{array}$ & $\begin{array}{c}\text { \% hojas } \\
\text { atacadas por } \\
\text { herbivoría }\end{array}$ & $\begin{array}{l}\text { \% área perdida } \\
\text { por herbivoría }\end{array}$ & $\begin{array}{c}\% \text { hojas dañadas } \\
\text { por factores } \\
\text { de estrés }\end{array}$ & $\begin{array}{l}\text { \% área } \\
\text { dañada }\end{array}$ \\
\hline & 180 & 1 & 1201 & $87(2.77) \mathrm{a}$ & $10.5(1.93) \mathrm{a}$ & - & - \\
\hline \multicolumn{8}{|c|}{ Q. pyrenaica } \\
\hline & 198 & 1 & 1947 & $88(2.80) \mathrm{a}$ & $8.86(0.73) a b$ & - & - \\
\hline \multicolumn{8}{|l|}{ Q. faginea } \\
\hline & 390 & 1 & 2685 & $52(6.05) \mathrm{b}$ & $7.10(0.97) b c$ & $53(4.82) b$ & $21(4.43) b c$ \\
\hline Q. suber & & 2 & 477 & $46(5.66)) \mathrm{b}$ & $7.18(0.78) b c$ & $87(3.80)$ a & $36(2.63) \mathrm{a}$ \\
\hline \multirow{3}{*}{ Q. ilex } & 772 & 1 & 4470 & $37(3.93) \mathrm{c}$ & $6.24(0.52) \mathrm{c}$ & $20(2.93) \mathrm{c}$ & $17(4.95) \mathrm{c}$ \\
\hline & & 2 & 4289 & $32(3.43) \mathrm{c}$ & $5.83(0.99) \mathrm{c}$ & $50(4.85) b$ & $25(5.02) \mathrm{abc}$ \\
\hline & & 3 & 756 & $34(4.00) \mathrm{c}$ & $6.02(0.77) \mathrm{c}$ & $65(6.40) \mathrm{ab}$ & $31(4.16) \mathrm{ab}$ \\
\hline
\end{tabular}

* Los datos de Longevidad foliar media para cada especie han sido tomados de Mediavilla y Escudero (2003a)

El análisis de los rasgos característicos de las distintos tipos foliares revela que, entre las hojas sanas de nuestras especies, se aprecia un incremento en LMA y espesor con el incremento de la longevidad foliar, presentando, para una misma clase de edad, $Q$. ilex valores superiores a los de $Q$. suber, y en ambas perennifolias más altos que los de las caducifolias (Tab. 2). En relación al tamaño, las hojas de Q. pyrenaica superan ampliamente al resto, entre las cuales las diferencias son más reducidas. 
Tabla 2. Diferencias en las características que, a nivel de una misma especie y clase de edad en las perennifolias (valor medio, SE entre paréntesis, $\mathrm{n}=50$ ), muestran las distintas categorías de hojas establecidas en función de los daños. Letras distintas para cada rasgo en cada especie y clase de edad reflejan diferencias significativas entre categorías para $\mathrm{P}<0.05$.

\begin{tabular}{|c|c|c|c|c|c|}
\hline Especie & Categoría & Edad & área/hoja $\left(\mathrm{cm}^{2}\right)$ & Espesor $(\mu \mathrm{m})$ & LMA $\left(\mathrm{gm}^{-2}\right)$ \\
\hline \multirow[t]{2}{*}{ Q. pyrenaica } & Sanas & 1 & $17.5(2.91) b$ & $161(2.47) \mathrm{a}$ & $135(3.94) \mathrm{a}$ \\
\hline & Herbivoría & 1 & $24.0(2.84) \mathrm{a}$ & $136(2.84) b$ & $113(4.43) b$ \\
\hline \multirow[t]{2}{*}{ Q. faginea } & Sanas & 1 & $6.63(0.36)$ a & $171(2.40) \mathrm{a}$ & $140(2.65) \mathrm{a}$ \\
\hline & Herbivoría & 1 & $5.81(0.56) \mathrm{a}$ & $160(2.59) \mathrm{b}$ & $126(2.53) \mathrm{b}$ \\
\hline \multirow[t]{6}{*}{ Q. suber } & Sanas & 1 & $4.82(0.48) \mathrm{a}$ & $238(3.12) \mathrm{a}$ & $172(2.59) \mathrm{a}$ \\
\hline & Dañadas por estrés & 1 & $5.46(0.63) \mathrm{a}$ & $22083.34) \mathrm{b}$ & $168(2.95) \mathrm{ab}$ \\
\hline & Herbivoría & 1 & $4.86(0.36) \mathrm{a}$ & $206(9.80) \mathrm{b}$ & $163(2.79) \mathrm{b}$ \\
\hline & Sanas & 2 & $2.54(0.34) \mathrm{a}$ & $259(5.21) \mathrm{a}$ & $189(3.06) \mathrm{a}$ \\
\hline & Dañadas por estrés & 2 & $3.93(0.28) \mathrm{a}$ & $243(4.93) \mathrm{a}$ & $180(2.02) \mathrm{b}$ \\
\hline & Herbivoría & 2 & $3.48(0.97) \mathrm{a}$ & $215(2.50) \mathrm{b}$ & $172(1.50) \mathrm{c}$ \\
\hline \multirow[t]{6}{*}{ Q. ilex } & Sanas & 1 & $3.11(0.31) \mathrm{a}$ & 278 (2.79) a & $240(5.01) \mathrm{a}$ \\
\hline & Dañadas por estrés & 1 & $2.98(0.32) \mathrm{a}$ & $264(5.07) b$ & $235(8.28) \mathrm{ab}$ \\
\hline & Herbivoría & 1 & $3.41(0.59)$ a & $252(3.86) b$ & $224(4.28) b$ \\
\hline & Sanas & 2 & $2.13(0.18) \mathrm{a}$ & $283(4.83) \mathrm{a}$ & $248(2.66) \mathrm{a}$ \\
\hline & Dañadas por estrés & 2 & $2.24(0.18) \mathrm{a}$ & 273 (4.87) a & $244(4.25) \mathrm{a}$ \\
\hline & Herbivoría & 2 & $1.88(0.14) \mathrm{a}$ & $265(3.61) b$ & $240(4.21) \mathrm{a}$ \\
\hline
\end{tabular}

Cuando efectuamos el análisis de regresión para comprobar si estas diferencias en los rasgos foliares pudieran relacionarse con los niveles de daños por herbivoría, no obtuvimos relación entre el tamaño de las hojas y la proporción de hojas dañadas (Tab. 3). La relación que sí se obtenía entre el área foliar y el porcentaje de área consumida por hoja se debe simplemente al hecho de que las hojas de Q. pyrenaica, como ya hemos comentado muy superiores en tamaño al resto, son también las más depredadas, desapareciendo la significación cuando se excluía de la regresión a esta especie. En principio podría pensarse que este resultado podría atribuirse al hecho de que el número de tipos de hoja incluidos en las regresiones, entre las cuales además no existen en algunos casos diferencias en los niveles de herbivoría (como ocurre entre las distintas cohortes de las perennifolias) es lo suficientemente reducido como para permitir arrojar significación en las relaciones. Sin embargo, con ese mismo tamaño de muestra sí se obtienen relaciones altamente significativas con el espesor y 
peso por unidad de superficie foliar, revelando nuestros datos que, a nivel de las distintas especies, el consumo es más alto en aquellas con hojas más finas y ligeras (Tab. 3). Nuestros resultados sugieren entonces que, al margen del efecto que otras características foliares puedan ejercer, como diferencias en composición química, también los rasgos que confieren consistencia, LMA y espesor, condicionan los niveles de herbivoría, permitiendo a las hojas mayor duración. Y esta relación entre el espesor y LMA de las hojas y los niveles de consumo por herbívoros parece observarse incluso a nivel intraespecífíco. Así, para una misma especie y clase de edad, no existían en general diferencias significativas entre el área de las hojas dañadas y las intactas, pero sí en LMA y espesor, que resultaban inferiores en las hojas dañadas en prácticamente todos los casos (Tab. 2).

Tabla 3. Resultados del análisis de regresión relacionando las características de las hojas dañadas por herbivoría (en valor medio para cada especie y clase de edad, $\mathrm{n}=7$ ) con los niveles de ataque por herbívoros (valor medio de $\%$ de hojas dañadas y \% de área perdida por hoja) detectados en los distintos tipos foliares considerados.

\begin{tabular}{cccc}
\hline Variable dependiente & Variable independiente & $\mathrm{R}^{2}$ & $\mathrm{P}$ \\
\hline \multirow{2}{*}{$\%$ hojas dañadas por herbivoría } & área/hoja $\left(\mathrm{cm}^{2}\right)$ & 0.53 & $\mathrm{NS}$ \\
& Espesor $(\mu \mathrm{m})$ & 0.92 & 0.0007 \\
& LMA $\left(\mathrm{gm}^{-2}\right)$ & 0.87 & 0.0024 \\
& & & \\
$\%$ área media perdida por hoja & área/hoja $\left(\mathrm{cm}^{2}\right)$ & 0.79 & $0.0077^{*}$ \\
& Espesor $(\mu \mathrm{m})$ & 0.92 & 0.0006 \\
& LMA $\left(\mathrm{gm}^{-2}\right)$ & 0.85 & 0.0032 \\
& $\%$ hojas dañadas por herbivoría & 0.91 & 0.0009 \\
\hline
\end{tabular}

* Esta relación pierde la significación cuando se excluyen las hojas de $Q$. pyrenaica, que superan ampliamente en tamaño al resto y son a la vez las más depredadas.

\subsection{Implicaciones de las diferencias en la intensidad de los daños sufridos por las hojas sobre su balance final de carbono.}

Una vez estimada la intensidad de los daños en las distintas especies, tratamos, a través de un sencillo modelo, de analizar sus implicaciones en términos de asimilación final de carbono. Para ello, calculamos en primer lugar el área que una misma cantidad de hojas de cada tipo (100) tendría asumiendo ausencia de daños y el que tendría teniendo en cuenta la intensidad de los daños obtenida en nuestro estudio (Tab. 4 y 5). Aunque no había diferencias significativas en la superficie individual entre hojas dañadas y no dañadas (Tab. 2), probablemente porque el porcentaje de superficie perdida es inferior a la variabilidad existente dentro de la población de hojas, hemos asumido que el tamaño promedio de cada hoja dañada sería inferior al de la 
hoja sana en la proporción estimada a partir de los datos de la tabla 1. Para estimar después las pérdidas de asimilación debidas a las pérdidas prematuras de área fotosintética, recurrimos a los datos de asimilación neta total de las hojas, obtenidos en un estudio realizado en tres de las especies incluidas en el presente trabajo en la misma parcela (Del Río 2014).

En el caso de los daños por herbivoría (Tab. 4), empleamos los datos de asimilación neta total para las hojas de cada especie $\left(\mathrm{mol} \mathrm{m}^{-2}\right)$, independientemente de la contribución que a esa cifra total pudieran tener las hojas en cada uno de los años de su vida, puesto que, como hemos comentado, no existían diferencias en la intensidad de la herbivoría que sufren las hojas de las distintas clases de edad (Tab. 1). Es decir, en este caso, se desprende que las hojas sufren herbivoría en las fases iniciales de su vida, sin nuevos ataques a medida que envejecen. Por tanto, podemos aplicar las mismas pérdidas por herbivoría prácticamente desde el inicio de la vida de las hojas. Asumiendo entonces que las hojas sanas y atacadas muestran similar capacidad fotosintética en la superficie no dañada (ver Discusión), obtuvimos la asimilación correspondiente al área que mostrarían 100 hojas intactas de cada especie, y al área que mostrarían las mismas 100 hojas tras aplicar los niveles de herbivoría obtenidos para esa especie, simplemente multiplicando ese área por las cifras de asimilación total por unidad de área obtenidas de Del Río (2014). A partir de las cifras registradas en ambos supuestos, calculamos finalmente el porcentaje de descenso en la asimilación obtenido como consecuencia de las pérdidas de área por herbivoría respecto a las cifras que se obtendrían en ausencia de herbivoría (Tab. 4). Los resultados revelan que las pérdidas de asimilación total debidas a las pérdidas de área por herbivoría muestran relación con la longevidad foliar, alcanzando valores hasta en torno a tres veces más altos en la especie caducifolia respecto a la de mayor longevidad foliar, $Q$. ilex, con valores intermedios en el alcornoque (Tab. 4).

Para analizar los costes asociados a las pérdidas de área por factores abióticos, desglosamos los datos de asimilación total conseguidos por las hojas de las tres es-

Tabla 4. Simulación del \% de descenso en la asimilación neta total de C conseguida por las hojas de tres de las especies de estudio debido a las pérdidas de área foliar atribuibles a los niveles de herbivoría detectados en cada caso, asumiendo similar asimilación en hojas sanas y atacadas.

\begin{tabular}{|c|c|c|c|c|c|c|}
\hline \multirow[b]{2}{*}{ Especie } & \multirow[b]{2}{*}{$\begin{array}{c}\text { Asimilación } \\
\text { neta }^{*} \\
\text { total/hoja } \\
\left(\mathrm{mol} \mathrm{m}^{-2}\right)\end{array}$} & \multicolumn{2}{|c|}{ Hojas no atacadas } & \multicolumn{2}{|c|}{ Hojas dañadas por herbivoría } & \multirow[b]{2}{*}{$\begin{array}{c}\% \text { descenso } \\
\text { asimilación total } \\
\text { Herbivoría - } \\
\text { No herbivoría }\end{array}$} \\
\hline & & $\begin{array}{c}\text { Area } \\
\text { total } \\
100 \text { hojas } \\
\left(\mathrm{m}^{2}\right)\end{array}$ & $\begin{array}{c}\text { Asimilación } \\
\text { neta } \\
\text { total } 100 \text { hojas } \\
(\mathrm{mol})\end{array}$ & $\begin{array}{c}\text { Área } \\
\text { total } \\
100 \text { hojas } \\
\left(\mathrm{m}^{2}\right)\end{array}$ & $\begin{array}{l}\text { Asimilación } \\
\text { neta } \\
\text { total } 100 \text { hojas } \\
\quad(\mathrm{mol})\end{array}$ & \\
\hline Q. faginea & 69 & 0.0663 & 4.57 & 0.0618 & 4.26 & 6.78 \\
\hline Q. suber & 65 & 0.0482 & 3.13 & 0.0464 & 3.02 & 3.51 \\
\hline Q. ilex & 132 & 0.0311 & 4.11 & 0.0304 & 4.01 & 2.43 \\
\hline
\end{tabular}

* Los datos de asimilación neta total a lo largo de la vida de las hojas fueron tomados de Del Río (2014). 
pecies en los distintos años a lo largo de los que transcurre su vida. Para ello recurrimos de nuevo al estudio de Del Río (2014), en el que se estimó la contribución porcentual de cada clase de edad foliar en las dos perennifolias al valor final de asimilación (primera columna de la Tabla 5). En este caso, a diferencia de lo que ocurría con la herbivoría, resulta imprescindible separar las distintas clases de edad, puesto que, como vimos (Tab. 1), los daños en las hojas se incrementan significativamente a medida que estas envejecen y asciende el tiempo de exposición a los diversos rigores climáticos. Contrariamente también a lo que ocurre en el caso de la herbivoría, los daños por estrés abiótico no se producen al inicio de la vida de las hojas. Los datos de área total mostrados en la Tabla 5 para las hojas dañadas de cada clase de edad corresponden a las pérdidas que se registrarían al final de cada periodo anual considerado, pero estos daños pueden aparecer como respuesta al rigor climático en cualquier momento del periodo anual. Puesto que en este estudio desconocemos el momento exacto en que se registran los daños para cada clase de edad, asumimos para simplificar que se producen en el punto medio de cada periodo anual de vida de la hoja. Por tanto las cifras de asimilación de las hojas dañadas de edad 1 se han obtenido asumiendo que durante la primera mitad del año están intactas y asignando las pérdidas

Tabla 5. Simulación del \% de descenso en la asimilación neta total de carbono conseguida por las hojas de tres de las especies de estudio debido a las pérdidas de área foliar atribuibles a los daños por factores de estrés detectados en nuestro estudio en las mismas especies, asumiendo similar asimilación en hojas sanas y en la superficie sana de las hojas dañadas.

\begin{tabular}{|c|c|c|c|c|c|c|c|}
\hline \multirow[b]{2}{*}{ Especie } & \multirow[b]{2}{*}{$\begin{array}{l}\text { Edad } \\
\text { foliar }\end{array}$} & \multirow[b]{2}{*}{$\begin{array}{l}\text { Asimilación } \\
\text { neta }^{*} \\
\text { total/hoja } \\
\left(\mathrm{mol} \mathrm{m}^{-2}\right)\end{array}$} & \multicolumn{2}{|c|}{ Hojas no dañadas } & \multicolumn{2}{|c|}{ Hojas dañadas por estrés abiótico } & \multirow[b]{2}{*}{$\begin{array}{c}\% \text { descenso } \\
\text { asimilación } \\
\text { Daños - } \\
\text { No daños }\end{array}$} \\
\hline & & & $\begin{array}{c}\text { Area } \\
\text { total } \\
100 \text { hojas } \\
\left(\mathrm{m}^{2}\right)\end{array}$ & $\begin{array}{l}\text { Asimilación } \\
\text { neta } \\
100 \text { hojas } \\
(\mathrm{mol})\end{array}$ & $\begin{array}{c}\text { Área } \\
\text { total }^{* *} \\
100 \text { hojas }\left(\mathrm{m}^{2}\right)\end{array}$ & $\begin{array}{c}\begin{array}{c}\text { Asimilación } \\
\text { neta*** }^{* * *}\end{array} \\
100 \text { hojas (mol) }\end{array}$ & \\
\hline \multirow[t]{2}{*}{ Q. suber } & $\begin{array}{l}1 \\
2\end{array}$ & $\begin{array}{l}54.6(84 \%) \\
10.4(16 \%)\end{array}$ & $\begin{array}{l}0.0482 \\
0.0254\end{array}$ & $\begin{array}{l}2.63 \\
0.26\end{array}$ & $\begin{array}{l}0.0428 \\
0.0174\end{array}$ & $\begin{array}{l}2.48 \\
0.19\end{array}$ & \\
\hline & & Total $=65$ & & Total $=2.89$ & & 2.67 & 7.61 \\
\hline \multirow[t]{2}{*}{ Q. ilex } & $\begin{array}{l}1 \\
2 \\
3\end{array}$ & $\begin{array}{l}62.0(47 \%) \\
56.8(43 \%) \\
13.2(10 \%)\end{array}$ & $\begin{array}{l}0.0311 \\
0.0213 \\
0.0195\end{array}$ & $\begin{array}{l}1.93 \\
1.21 \\
0.26\end{array}$ & $\begin{array}{l}0.0300 \\
0.0186 \\
0.0156\end{array}$ & $\begin{array}{l}1.89 \\
1.09 \\
0.19\end{array}$ & \\
\hline & & Total $=132$ & & Total $=3.40$ & & Total $=3.17$ & 6.76 \\
\hline
\end{tabular}

* Los datos de asimilación neta total y la contribución de las hojas durante cada uno de los años de su vida a ese valor total fueron tomados de Del Río (2014).

** Las cifras revelan las pérdidas de área que registrarían las hojas al final del periodo anual considerado.

*** La asimilación neta de las hojas dañadas se estimó asumiendo que los daños en cada clase de edad se producen a mitad de ese año de su vida. 
de área a la segunda mitad del año. Del mismo modo, para las hojas de la segunda cohorte las cifras de asimilación se han obtenido asumiendo las pérdidas de área que mostraban durante su primer año de vida más la mitad de las pérdidas registradas al final de su segundo año, puesto que consideramos que se producen a mitad de este segundo año. Cuando estimamos el descenso final en la asimilación neta total conseguida por las hojas dañadas respecto a las intactas comprobamos que este alcanza cifras muy superiores a los descensos en asimilación registrados por herbivoría en las dos especies.

\section{Discusión}

El estudio de las implicaciones de las diferencias en longevidad foliar sobre el rendimiento fotosintético y, por tanto, capacidad competitiva de las distintas especies ha centrado una parte muy importante de la investigación en ecofisiología (Westoby et al., 2000; Takashima et al., 2004). La mayor duración de las hojas de las especies perennifolias implica un mayor tiempo de exposición tanto a los herbívoros, como a los distintos factores de estrés climático, de modo que, para que puedan persistir, deben presentar atributos que les confieren protección y que implican mayores costes de construcción y menores tasas fotosintéticas, que podrían ser compensadas con el mayor tiempo del que disponen para revertir a la planta lo invertido en ellas. En las caducifolias, sin embargo, la menor duración de las hojas implica un tiempo limitado para fotosintetizar, lo que impone altas tasas instantáneas que sólo pueden ser conseguidas con menor peso por unidad de superficie y mayores contenidos de nutrientes, lo que implicaría menores costes de construcción, pero, en principio, les haría también más susceptibles frente a los rigores del clima y a los herbívoros (Wright and Cannon, 2001; Wright and Westoby, 2002). Todas estas diferencias deberían traducirse en diferencias en los daños que experimentan por distintos factores a lo largo de su vida.

Por lo que se refiere a la herbivoría, encontramos una relación inversa entre la intensidad de las pérdidas y la longevidad foliar. Tanto el porcentaje de hojas atacadas, como la fracción de área media perdida por hoja descienden a medida que se incrementa la duración media de las hojas de nuestras especies de estudio. Este resultado coincide con el de otros estudios que también han encontrado menores tasas de herbivoría en las especies perennifolias respecto a las caducifolias (Coley, 1988; Poorter et al., 2004; Pringle et al., 2011). Los datos de estos y otros autores sugieren además que los herbívoros seleccionan las hojas en función de su rigidez y digestibilidad más que de su valor nutritivo. En nuestro caso hemos obtenido una relación inversa altamente significativa entre LMA y el espesor medio de los distintos tipos de hoja con los niveles de herbivoría. Ambos rasgos, LMA y el espesor, se sabe que están positivamente correlacionados con la rigidez y dureza de las hojas (Reich et al., 1991), considerándose a menudo como una adaptación frente el ataque de los herbívoros (Kudo, 1996). Y estos resultados se repiten tanto a nivel interespecífico, como incluso a nivel de las hojas de una misma especie, correspondiendo en prácticamen- 
te todos los casos los mayores niveles de herbivoría a las hojas con menor espesor y LMA dentro de cada tipo analizado. En principio, estas diferencias sugieren que los herbívoros parecen seleccionar, por tanto, no sólo los árboles de aquellas especies con hojas menos rígidas y duras, sino a nivel de cada especie, aquellas hojas con menor LMA y espesor. Alternativamente, no podemos descartar la posibilidad de que el menor LMA de las hojas que han sufrido el ataque de los herbívoros se deba a un menor crecimiento en espesor motivado precisamente por el ataque y no a una selección previa por parte de los herbívoros. Sin embargo, lo cierto es que no había diferencias en la superficie total por hoja entre las dañadas y las intactas (Tab. 2), lo que sugiere que las pérdidas iniciales no afectaron al crecimiento posterior en área. No encontramos, sin embargo, respecto a la herbivoría, diferencias entre las distintas clases de edad presentes en las perennifolias. Este resultado se explica por el hecho de que la mayor parte de la herbivoría se sabe que ocurre en las fases iniciales del desarrollo de las hojas (Coley, 1983, 1988; Kudo, 1996), cuando poseen mayor concentración de nutrientes, menor densidad y menor contenido de fibras, que determinan mayor preferencia de los herbívoros. Los niveles de herbivoría parecen, por tanto, ser independientes del tiempo de exposición de las hojas a los herbívoros.

Respecto a los daños por factores abióticos, la rigidez de las hojas, medida a través del espesor y peso por unidad de superficie, también parece condicionar su intensidad. Aunque en este caso sólo disponemos de datos para las dos especies perennifolias, se aprecia claramente que tanto el porcentaje de hojas dañadas, como la proporción de área afectada resultaban superiores en la especie de hojas menos longevas, $Q$. suber, y con menor LMA y espesor foliar. Este resultado confirma de nuevo la importancia de los rasgos que confieren robustez a las hojas. Sin embargo, al contrario de lo que ocurría con los niveles de herbivoría, sí apreciamos un marcado incremento de los daños a través de las distintas cohortes foliares. La intensidad de los daños causados por factores abióticos se incrementa, como era de esperar, con la edad de las hojas $\mathrm{y}$, por tanto, a medida que lo hace el tiempo de exposición a estos distintos factores de estrés. Se plantea, entonces, un compromiso. Para incrementar la duración de sus hojas, las especies con mayor longevidad presentan rasgos que disminuyen la susceptibilidad a los daños por factores climáticos, pero, por otro lado, aumentar la duración de las hojas implica mayor intensidad de los daños a medida que las hojas duran más. Y la proporción de área dañada por factores abióticos alcanza cifras siempre superiores a los daños causados por herbivoría. Es posible entonces que las ventajas de una mayor longevidad en términos de pérdida de asimilación futura por pérdidas de área por herbivoría no lo sean tanto en términos de pérdidas por daños abióticos.

Para tratar de resolver esta cuestión elaboramos un sencillo modelo intentando determinar las pérdidas de asimilación debidas a pérdidas de área productiva tanto por factores bióticos (herbivoría), como abióticos. Nuestro modelo parte de la suposición de que tanto las hojas dañadas como las intactas presentan similar capacidad fotosintética. Es cierto que se han propuesto distintas respuestas compensatorias a las pérdidas de área, entre las cuales figuran el incremento en las tasas fotosintéticas en hojas atacadas (Retuerto et al. 2004), o bien en hojas sanas para ganar más carbono y compensar las pérdidas de las hojas dañadas (Kudo 1996), o un mayor desarrollo 
del área foliar en las hojas dañadas (Strauss \& Agrawal 1999, Hattori et al. 2004). Sin embargo, también varios estudios han mostrado ausencia de diferencias en la tasa fotosintética entre ambos tipos de hojas (Meyer \& Whitlow 1992, Chen et al. 2001). Tampoco un estudio llevado a cabo por nuestro equipo con una de las especies incluidas en el presente trabajo reveló diferencias en las tasas fotosintéticas en hojas sanas y atacadas (Silla et al. 2008). Asumiendo entonces que tanto las hojas sanas como las dañadas presentan similar capacidad fotosintética, encontramos que el descenso en el rendimiento final debido a las pérdidas de área por herbivoría se reduce con el incremento de la longevidad foliar. Así, la especie caducifolia ve reducida su asimilación final de carbono en torno a tres veces más que en la especie de hojas más longevas, mostrando $Q$. suber valores intermedios. Sin embargo, cuando analizamos las pérdidas de asimilación debidas a pérdidas de área fotosintética por factores de éstres abiótico comprobamos que las cifras ascienden notablemente, debido fundamentalmente al descenso en asimilación que muestran las hojas de mayor edad, más elevado en las cohortes más viejas. En principio, podríamos concluir entonces que las ventajas de una mayor longevidad foliar reduciendo el ataque de los herbívoros se ven contrarrestadas por el incremento en los daños que sufren las hojas a medida que se incrementa su duración y su exposición a los rigores climáticos, teniendo en cuenta además que las pérdidas de asimilación por pérdidas de área debidas a factores abióticos superan a las cifras obtenidas por herbivoría.

Somos conscientes de que extraer conclusiones más precisas requiere de nuevos estudios que incorporen un mayor número de especies y permitan resolver cuestiones como las diferencias en las tasas de asimilación entre hojas sanas y dañadas o el momento exacto en que se producen los daños en las hojas de las distintas especies. Sin embargo, también es cierto que estudios de este tipo, aportando información sobre los daños por distintos factores en hojas de distinta duración, resultan imprescindibles para poder precisar adecuadamente la capacidad final de secuestro de carbono de las distintas especies.

\section{Agradecimientos}

Este trabajo ha recibido financiación del Ministerio de Ciencia e InnovaciónEU-FEDER (CGL2010-21187) y la Fundación Miguel Casado S José.

\section{Bibliografía}

Abràmoff, M.D., Magalhaes, P.J., Ram, S.J. 2004. Image processing with ImageJ. Biophotomics International 11, 36-42.

Aerts, R., 1995. The advantages of being evergreen. Trends Ecol. Evol 10, 402-407.

Chen, Z., Kolb, T.E., Clancy, K.M. 2001. Mechanisms of Douglas-fir resistance to western spruce budworm defoliation: bud burst phenology, photosynthetic compensation and growth rate. Tree Physiol. 21, 1159-1169. 
Coley, P.D., 1983. Herbivory and defensive characteristics of tree species in a lowland tropical forest. Ecol. Monogr. 53, 209-233.

Coley, P.D., 1988. Effects of plant growth rate and leaf Lifetime on the amount and type of anti-herbivore defense. Oecologia 74, 531-536.

Del Rio, T., 2014. Fotosíntesis o persistencia: implicaciones de la longevidad foliar sobre la fijación del carbono en especies leñosas en un ambiente estacional. Tesis Doctoral. Universidad de Salamanca.

Dewar, R.C., Franklin, O., Mäkelä, A., McMurtie, R.E., Valentine, H.T., 2009. Optimal function explains forest responses to global change. Bio. Sci. 59, 127-139.

Dorronsoro, D.F., 1992. Suelos. En: Gómez Gutiérrez, J.M. (ed.), El libro de las dehesas salmantinas. Junta de Castilla y León, Salamanca, pp. 71-124.

Falster, D.S., Reich, P.B., Ellsworth, D.S., Wright, I.J., Westoby, M., Oleksyn, J., Lee, T.D., 2011. Lifetime return on investment increases with leaf lifespan among 10 Australian woodland species. New Phytol. 193, 409-419.

Günthardt-Goerg, M.S., Vollenweider, P., 2007. Linking stress with macroscopic and microscopic leaf response in trees: new diagnostic perspectives. Environ. Pollut. 147, 467488.

Hattori, K., Ishida, T.A., Suzuki, M., Kimura, M.T., 2004. Differences in response to simulated herbivory between Quercus crispula and Quercus dentata. Ecol. Res. 19, 323-329.

Heberling, J.M., Fridley, J.D., 2012. Biogeographic constraints on the world-wide leaf economics spectrum. Global Ecol. Biogeog. 21, 1137-1146.

Kikuzawa, K., Lechowicz, M.J., 2006. Toward synthesis of relationships among leaf longevity, instantaneous photosynthetic rate, lifetime leaf carbon gain, and the gross primary production of forests. Am. Nat. vol. 168 No. 3.

Kudo, G., 1996. Herbovory pattern and induced responses to simulated herbivory in Quercus mongolica va grosseserrata. Ecol. Res. 11, 283-289.

Matsuki, S., Koike, T., 2006. Comparison of leaf life span, photosynthesis and defensive traits across seven species of deciduous broad-leaf tree seedlings. Ann. Bot. 97, 813-817.

Mediavilla, S., Escudero, A., 2003a. Photosynthetic capacity, integrated over the lifetime of a leaf, is predicted to be independent of leaf longevity in some tree species. New Phytol. 159, 203-211.

Mediavilla, S., Escudero, A., 2003b. Leaf life span differs from retention time of biomass and nutrients in the crowns of evergreen species. Funct. Ecol. 17, 541-548.

Meyer, G.A., Whitlow, T.H., 1992. Effects of leaf and sap feeding insects on photosynthetic rates of goldenrod. Oecologia 92, 480-489.

Poorter, L., van de Plassche, M., Willems, S., Boot, G.A., 2004. Leaf traits and herbivory rates of tropical tree species differing in successional status. Plant Biol. 6, 1-9.

Pringle, E.G., Adams, R.I., Broadbent, E., Busby, P.E., Donatti, C.I., Kurten, E.L., Renton, K., Dirzo, R., 2011. Distints leaf-trait syndromes of evergreen and deciduous trees in a seasonally dry tropical forest. Biotropica 43, 299-308.

Reich, P.B., Kloeppel, B.D., Ellsworth, D.S., Walters, M.B., 1995. Different photosynthesisnitrogen relations in deciduous hardwoods and evergreen coniferous tree species. Oecologia 104, 24-30.

Reich, P.B., Uhl, C., Walters, M.B., Ellworth, D.S., 1991. Leaf lifespan as a determinant of leaf structure and function among 23 Amazonian tree species. Oecologia 86, 16-24. 
Retuerto, R., Fernández-Lema, B., Rodríguez-Roiloa, S., Obeso, J.R., 2004. Increased photosynthetic performance in Holly trees infested by scale insects. Funct. Ecol. 18, 664-669.

Shipley, B., Lechowicz, M.J., Wright, I., Reich, P.B., 2006. Fundamental tradeoffs generating the worldwide leaf economics spectrum. Ecology 87, 535-541.

Silla, F., Fleury, M., Mediavilla, S., Escudero, A., 2008. Effects of simulated herbivory on photosynthesis and N resorption efficiency in Quercus pyrenaica Willd. Saplings. Trees 22, 785-793.

Strauss, S.Y., Agrawal, A.A., 1999. The ecology and evolution of plant tolerance to herbivory. Trens Ecol. Evol. 14, 179-185.

Takashima, T., Hikosaka, K., Hirose, T., 2004. Photosynthesis or persistence: nitrogen allocation in leaves of evergreen and deciduous Quercus species. Plant Cell Environ. 27, 1047-1054.

Vaz, M., Maroco, J., Ribeiro, N., Gazarini, L.C., Pereira, J.S., Chaves, M.M., 2011. Leaf-level responses to light in two co-occurring Quercus (Quercus ilex and Quercus suber): leaf structure, chemical composition and photosynthesis. Agrof. Syst. 82, 173-181.

Vollenweider, P., Günthardt-Goerg, M.S., 2005. Diagnosis of abiotic and biotic stress factors using the visible symptoms in foliage. Environ. Pollut. 137, 455-465.

Warren, C.R., Adams, M.A., 2000. Trade-offs between the persistence of foliage and productivity in two Pinus species. Oecologia 124, 487-494.

Westoby, M., Warton, D., Reich, P.B., 2000. The time value of leaf area. Am. Nat. 155, 649656.

Wright, I.J., Cannon, K., 2001. Relationships between leaf lifespan and structural defences in a low nutrient, sclerophyll flora. Funct. Ecol. 15, 351-359.

Wright, I.J., Reich, P.B., Westoby, M., Ackerly, D.D., Baruch, Z., Bongers, F., CavenderBares, J., Chapin, F.S., Cornelissen, J.H.C., Diemer, M., Flexas, J., Garnier, E., Groom, P.K., Gulias, J., Hikosaka, K., Lamont, B.B., Lee, T., Lusk, C., Midgley, J.J., Navas, M.L., Niinemets, U., Oleksin, J., Osada, N., Poorter, H., Poot, P., Prior, L., Pyankov, V.I., Roumet, C., Thomas, S.C., et al., 2004. The world-wide leaf economics spectrum. Nature 428, 821-827.

Wright, I.J., Westoby, M., 2002. Leaves at low versus high rainfall: coordination of structure, lifespan and physiology. New Phytol. 155, 403-416. 
